\title{
Implementation of Smart Pot System using USB Plug-in Sensor
}

\author{
Yeong-Keun Lee, Koo-Rack Park, Dong-Hyun-Kim
}

\begin{abstract}
Since the IoT based technologies were applied to various areas, people's living has been more convenient and efficient. In present society, the number of one-person households is on the constant rise, and the industrial advancement causes their psychological alienation more. In the circumstance, a variety of methods to recover their psychological stability try to be found. More people regain their mental stability in the way of raising plants. Therefore, this study proposes the smart pot system using a USB plug-in sensor. In this study was implemented the smart pot system that is able to check a plant's status on the basis of accurate data, such as plant growth information and environmental information, at any times and any places, to control and maintain the environment of growth, to monitor environmental information by setting and collecting automatically such data as temperature, humidity, solar radiation quantity, $\mathrm{CO} 2$, and growth conditions, to detect abnormal situations through human body monitoring and fire monitoring of indoor conditions, and to prevent dangerous circumstances. In the market, there are already home pot products to which IoT technology is applied. However, most of the smart pots fail to take into account species of plants and are recommended to be used for particular plants only. The proposed system is the USB plug-in based smart pot system capable of monitoring and controlling plant growth, supporting human relationship, and implementing user-customized functions by easily expanding sensors which are able to notice indoor emergency and disaster situations in everyday life. It also can add the function of meeting an emotional need by changing a LED color when people come close to their pet plants. The proposed system is expected to help many people growing plants easily realize plant growing conditions and indoor situations and improve their psychological stability.
\end{abstract}

Keywords : IOT, Smart Flower Pot, Temperature, Humidity, Automatic Watering, Plug-in.

\section{INTRODUCTION}

$W_{\text {ith the rapid development of the IT industry, smart }}$ products have been released in various areas. Since IoT (Internet of Things) technology, which makes it possible to collect and exchange a variety of information through physical devices with built-in sensor network and software, was developed [1], they have been applied to industrial fields and home products based on IoT technology have been more commercialized [2]

Revised Manuscript Received on July 22, 2019.

Yeong-Keun Lee, Dept. of Computer Engineering, Kongju National University, Cheonan, Rep. of Korea. Email: johnyklee1@ naver.com

Koo-Rack Park*, Dept. of Computer Science \& Engineering, Kongju National University, Cheonan, Rep. of Korea. Email: ecgrpark@kongju.ac.kr

Dong-Hyun Kim, Dept. of Computer Engineering, Kongju National University, Cheonan, Rep. of Korea. Email: dhkim977@ naver.com

In particular, the IoT technology is applied to plant pots which are used at home for various purposes, so that IoT based plant pots sell in the market. However, most of the smart pots fail to take into account species of plants and are recommended to be used for particular plants only. With the change and development of overall social environments like industrial advancement, the number of one-person households is on the constant increase.

For various reasons, such as fatigue in busy urban life, environmental issues like fine dust, and a changing lifestyle, people try to find diverse ways to recover their psychological stability. In particular, more people grow plants to regain their mental stability in the way of looking at flowers and fruits of their plants. In the US, even young people are more interested in growing plants since their satisfaction is high in terms of cost. According to the report of New York Times, a great deal of people who newly enter the plant growth market was millennium generation [3].

In this aspect, it is necessary to research the system platform that can expand or reduce to a customized system by meeting different user needs in any circumstances.

Given that pots are mostly in the same position, this study proposes the plug-in based smart pot system that provides the security function of detecting a fire and a trespasser and monitoring smooth growth of plants with the use of various sensors, and of the control function for LED light of smart pots with the use of a ultrasonic sensor, beyond the function of supplying water to plants with the simple use of sensor information. The proposed system is the USB plug-in based customized smart pot system that can control pots through remote smart devices like smartphone and PC.

\section{RELATED WORKS}

In order to implement the customized smart pot system, relevant studies on smart pots and smart farms were analyzed so as to look into the contents of plant growth.

\section{A. Smart pot}

With the rapid development of sensor technology, communication technology, and digital technology, effective methods of collecting environmental information have been provided [4]-[6]. There are some relevant studies on smart pots. In the study [2], wheels were attached to a pot and the pot robot with various sensors and a control board was researched. In the study [7], RIFD tags were attached to garden paths so that a robot transported water along the given paths, and 
water was supplied autonomously through sensor detection of temperature and humidity.

The study [8] was conducted on the remote control smart pot system capable of measuring the mediating variables of plant growth through temperature \& humidity sensor photosensitive resistance. The study [9] was conducted on the auto light-seeking pot that is able to collect flower environment information such as temperature, humidity, intensity of light, and a water level, to display the information on LCD screen, and to make automatic water supply and light seeking on the basis of feedback information. However, most studies focused on plant growth.

\section{B. Smart farm}

There are some previous studies on smart farms as follows. In order to solve the interaction problem of IoT, the study [10] was conducted on the interaction with the smart garden device with temperature \& humidity sensors and a smartphone in several scenarios of interaction between a user and smart objects in smart space. The study [11] focused on the embedded controller based automated nutrient supply and pollutant removal in hydroponics. In order to solve the problem that farmers have due to their lack of knowledge of cultivation, the study [12] was conducted on plant pot modulation that includes the motor of supplying water in interaction with sensors of water, nutrients, and sunlight, and LED replacing solar energy.

The study [13] was conducted on the prototype that is able to display information on PC and mobile device through the humidity detection sensor and IoT based sensor for mushroom farms, and to automatically control spring coolers and mist pumps. In the analysis on these relevant works, it was possible to understand the process of collecting plant growth data with the use of IoT technology and the platform and networking essential to the devices to control.

Most of previous studies on smart farms focused on the system that provides a particular environment for a particular plant in a fixed way.

\section{THE PROPOSED SYSTEM}

To implement a smart pot, this study proposes the model for controlling water supply, intensity of light, temperature \& humidity, and indoor conditions which are essential to plant growth.

[Fig. 1] illustrates the system architecture of the proposed model which has three parts.

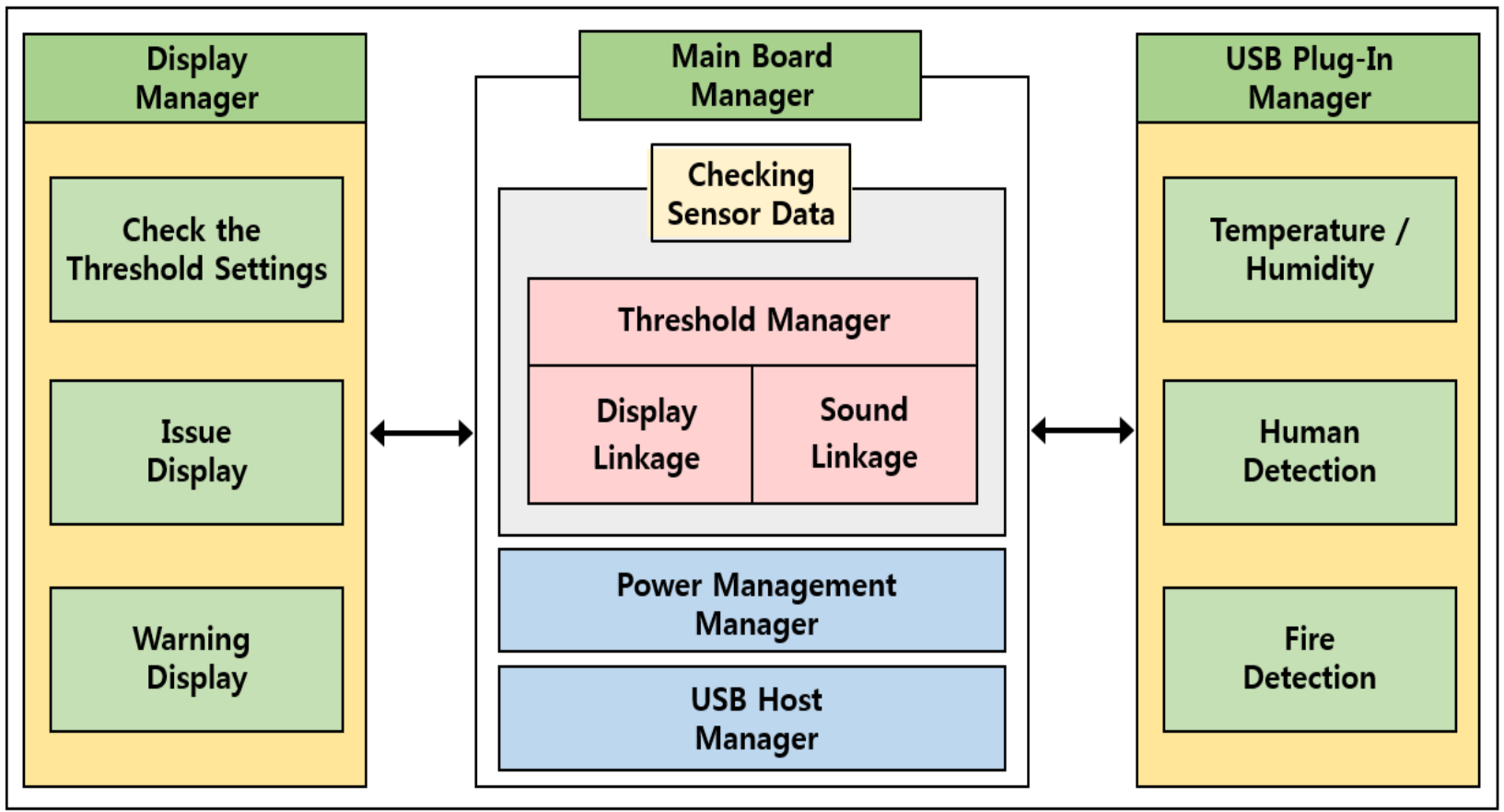

Fig. 1. System Architecture

Firstly, Main Board Manager is able to check input data of various sensors, to manage power, and to control the USB Host for sensor connection. In addition, it is able to display a variety of connection data on LCD for user check, and to make an alarm in sound connection.

Secondly, USB Plug-in Manager enables a user to select, install, and control proper sensors, such as the basic sensor for monitoring moisture and sunlight for plant growth, the fire detection sensor and gas leakage sensor for monitoring indoor status, and the human detection sensor for monitoring thieves.

Thirdly, Display Manager is able to display a text or a warning on LCD in order for a user to check the threshold value of each sensor.

[Fig. 2] shows the process of recognizing various types of sensors, which consists of four steps.

Firstly, there are various types of sensors, since they are based on USB. The power of these sensors is supplied by USB Hub.

Secondly, to recognize various types of sensors, power is supplied to the sensors through USB Hub, and thereby sensor data is collected. 
Thirdly, to check if the input sensor value is humidity data, the process $\mathrm{A}$ is executed. In the process $\mathrm{A}$, a threshold value is checked, and then is displayed on LCD.

Fourthly, to check if the input sensor is human detection sensor data, the process B is executed. Fifthly, different processes are executed depending on sensor types.

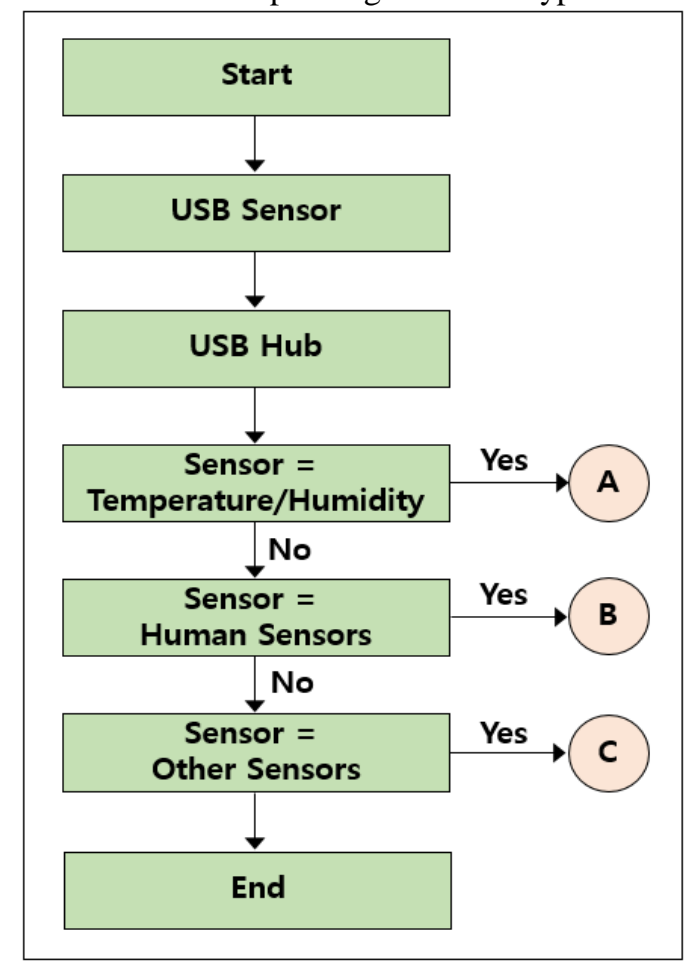

Fig. 2. Sensor-type Recognition Process

[Fig. 3] presents the sensor-type recognition process which comprises 4 steps.

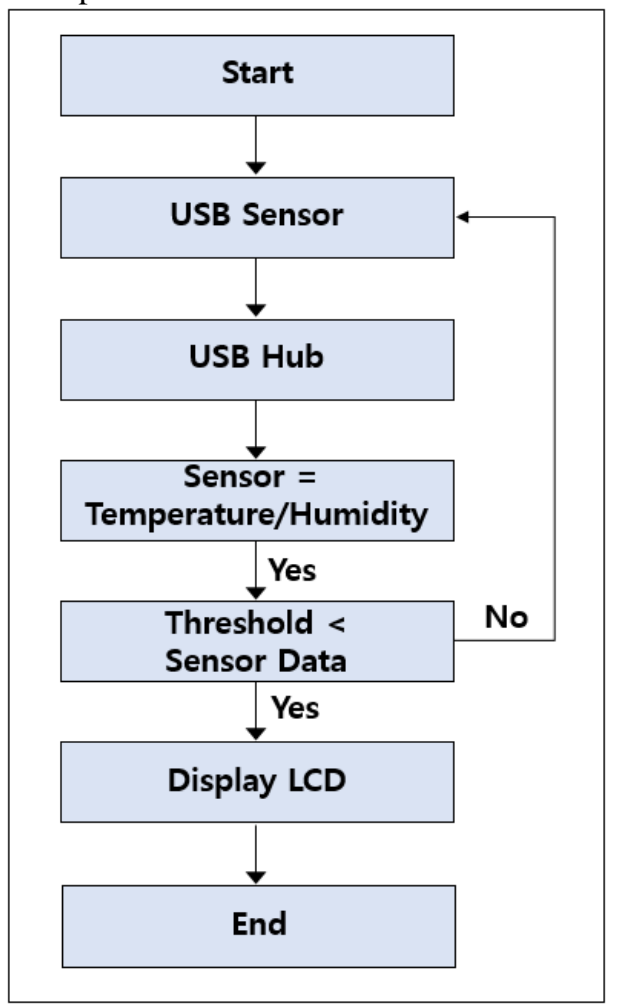

Fig. 3. Sensor Recognition Execution Process

A process is executed after a type of sensor is recognized through USB Hub. In the process, humidity status can be displayed on LCD. If a humidity USB sensor is connected to USB Hub, the process makes it possible to check if the input sensor data is humidity data, and then to compare the set threshold value with the data if it is judged to be humidity data. If the input sensor data is higher than the threshold value, an alarm message is displayed on LCD.

[Fig. 4] shows a part of the algorithm of recognizing a type of USB Plug-in sensor.

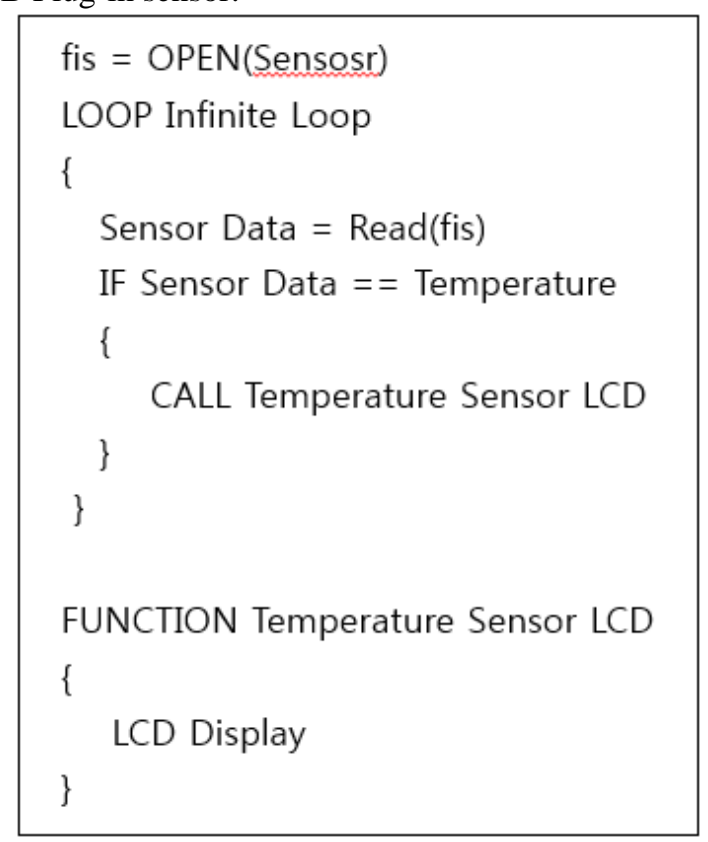

Fig. 4. Sensor Type Recognition Algorithm

A type of sensor is recognized in the following procedure:

First, in the 'fis $=\mathrm{OPEN}$ (Sensor)' statement, a sensor is connected and opened.

Secondly, in the LOOP statement, the loop is repeated infinitely.

Thirdly, in the 'Sensor Data = READ(fis)' statement, the data collected from the sensor is read.

Fourthly, in the 'IF' statement, whether the sensor value is temperature data is checked.

Fifthly, in the 'CALL' statement, a threshold value is checked, and then the function of LCD display is called.

Sixthly, if the input temperature value is larger than the threshold value, a warning and check message is displayed in a text form on LCD.

\section{RESULTS AND DISCUSSION}

[Fig. 5] illustrates the developed components to implement the proposed model.

To implement the functions of the proposed model, "Arduino Uno R3" was used for the main board, and a breadboard was used to expand additional ports.

To give a notice to a user when a sensor value reaches its threshold value, 16x2 LCD module was used. For power supply, a 6xAA battery holder was used. 


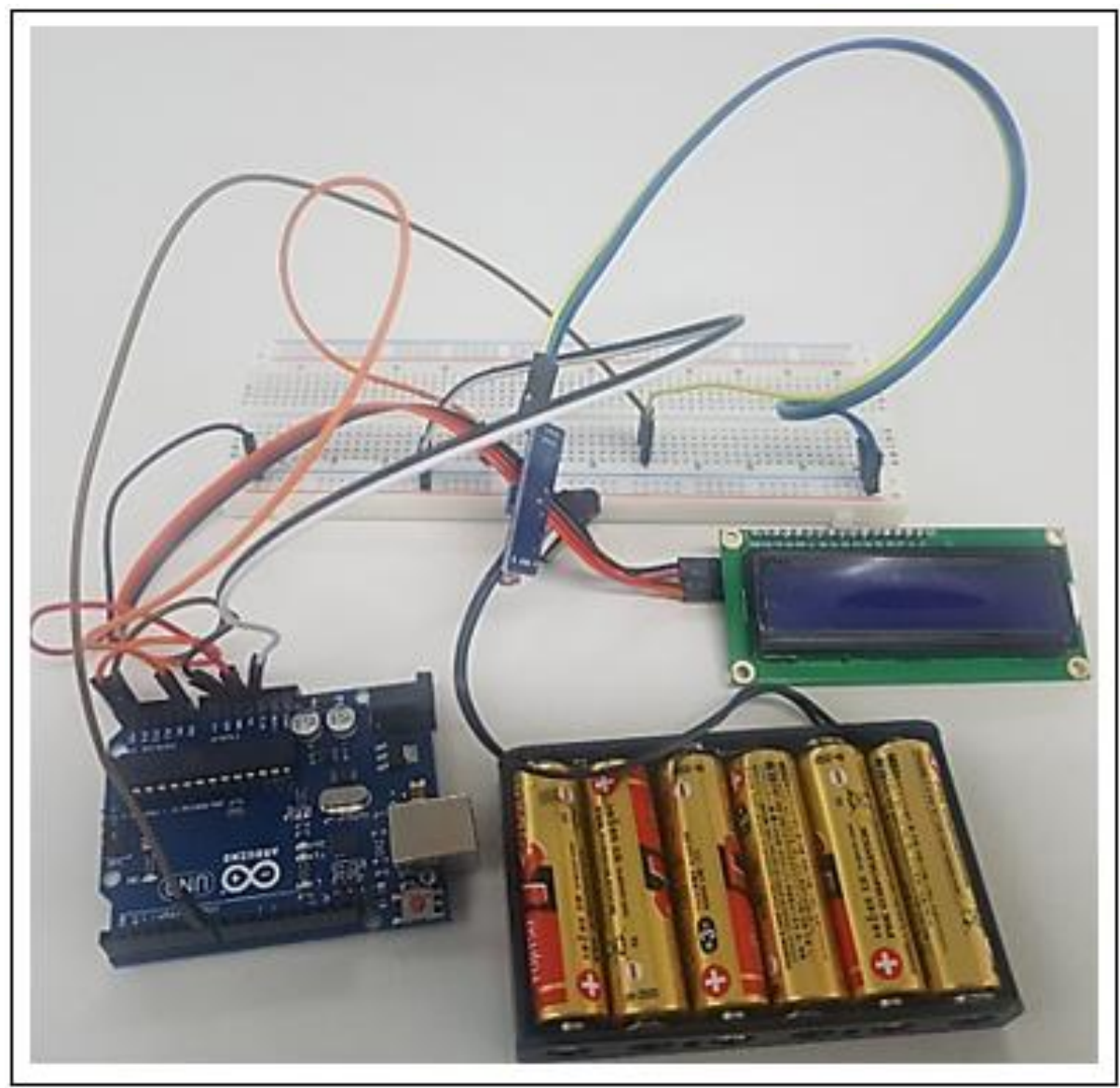

Fig. 5. Developed Components

[Fig. 6] shows the LCD display for noticing issues.

Figure (a) presents the display of humidity sensor data, and the threshold value of humidity sensor is set to ' 600 '. If water needs to be supplied after the humidity of a flower pot is measured, a message is displayed on LCD.

Figure (b) presents the illumination detection sensor data. If a flower pot fails to receive sunlight for 6 days in a row, a message is displayed on LCD.

Figure (c) presents the human detection sensor data. If a human is detected, a message is displayed on LCD. With the use of the human detection sensor, it is possible to monitor trespassers like a thief.

Figure (d) presents the fire detection sensor data. If a flame is detected, a message is displayed on LCD.

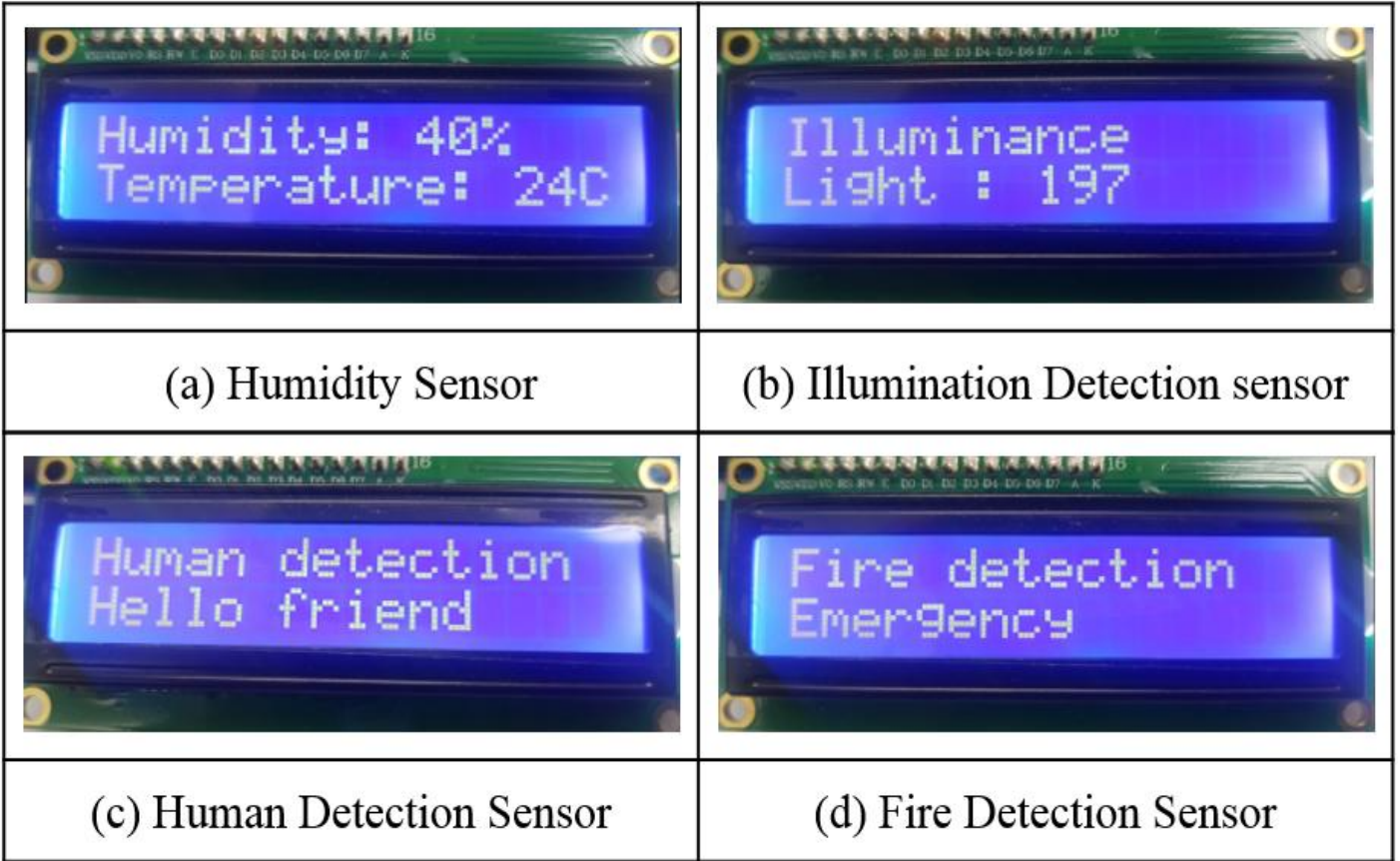

Fig. 6. Issue Notification LCD 
[Table I ] presents types of sensors which can be applied to the proposed model.

Table- I: Sensor Type \& Application

\begin{tabular}{|c|c|}
\hline Sensor Type & Application \\
\hline Sound detection sensor & $\begin{array}{c}\text { Detection of a dangerous } \\
\text { situation }\end{array}$ \\
\hline $\begin{array}{c}\text { Fine dust detection } \\
\text { sensor }\end{array}$ & $\begin{array}{l}\text { Detection of an indoor air } \\
\text { condition }\end{array}$ \\
\hline $\begin{array}{l}\text { Smoke \& flame detection } \\
\text { sensor }\end{array}$ & Detection of a fire \\
\hline Illumination sensor & $\begin{array}{l}\text { Judgment of the necessity of } \\
\text { sunlight }\end{array}$ \\
\hline Temperature sensor & $\begin{array}{c}\text { Maintenance of a proper indoor } \\
\text { temperature }\end{array}$ \\
\hline Soil humidity sensor & Judgment for watering plants \\
\hline Ultrasonic sensor & Judgment for joyfully greeting a \\
\hline
\end{tabular}

person who comes close to a smart pot

[Fig. 7] illustrates an example of test-product application. Figure (a) displays a message on LCD when a sensor data reaches its threshold value. Figure (b) means the device for power supply, has the 5Pin cable mostly used in smartphone, and is recharged with solar charger. Figure (c) is used to connect a USB sensor through USB Hub. In addition, if a USB port is needed additionally, it is possible to make a connection with the use of an external USB Hub. Figure (d) represents the main board which has main functions. Since the main board has a rechargeable battery, it is possible to use the smart pot in a place without electricity.

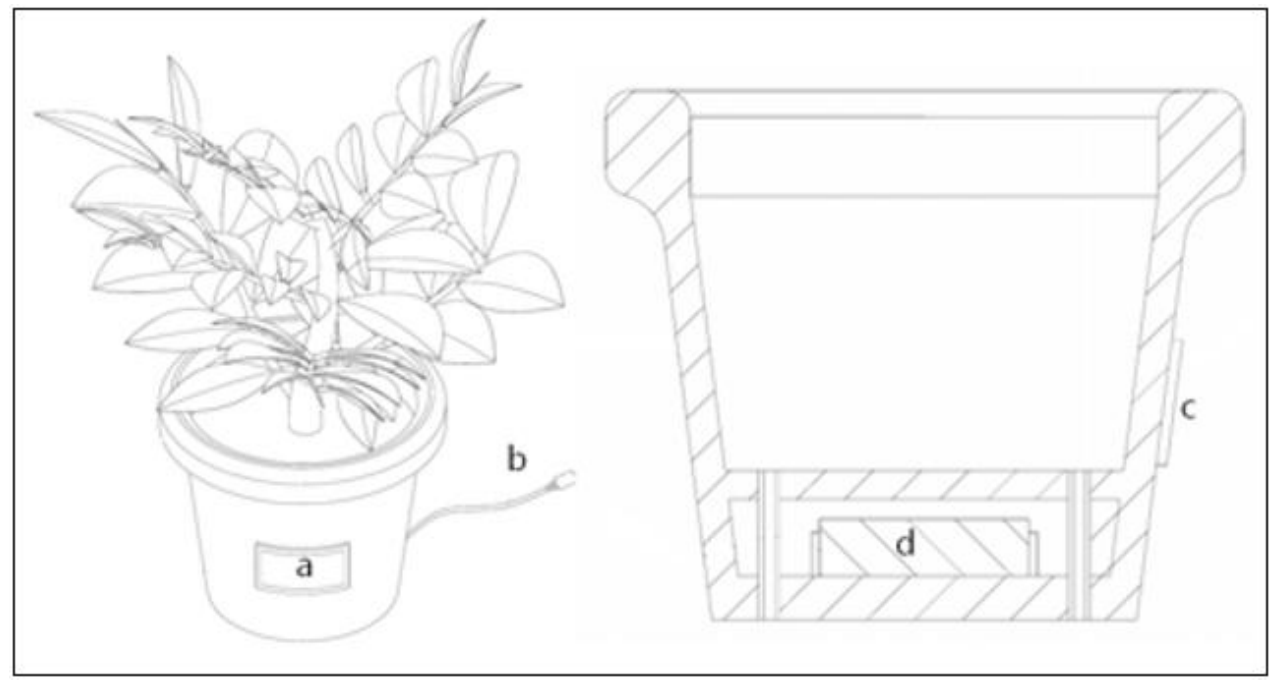

Fig. 7. An Example of Test-Product Applications

\section{CONCLUSION}

With the development of ICT, many types of smart pots are actively researched, and smart pots sell. In fact, there are various kinds of cultivation methods depending on plant types and characteristics. However, most smart pots in sales simply give an alarm when the problem of water status occurs, or provide simple monitoring function through mobile with the use of specified sensor settings. This study proposed the USB connectable plug-in smart pot system that makes it possible for users to set up necessary sensors directly and check them, and to detect an emergency situation at home through multiple sensors of smart pots. With the proposed system, it is possible to detect a flame or heat to monitor a fire and to check any trespasser through human detection sensor.

In addition, the system can provide the additional function of satisfying an emotional need by changing a color of LED when a pet plant cultivator comes close. In the future, it will be necessary to continuously research the platform of smart garden and smart farm that helps users who lack the knowledge of plant growth understand and predict plant growth conditions.

\section{REFERENCES}

1. Atzori, Luigi, Antonio Iera, and Giacomo Morabito, "The internet of things: A survey," Computer networks, vol. 54, no. 15, 2010, pp. 2787-2805. https://doi.org/10.1016/j.comnet.2010.05.010

2. Kawakami, A., Tsukada, K., Kambara, K., \& Siio, I., "Potpet: pet-like flowerpot robot," in Proceedings of the fifth international conference on Tangible, embedded, and embodied interaction. ACM New York, 2011, pp. 263-264. https://doi.org/10.1145/1935701.1935755

3. The New York Times, Plant-Loving Millennials at Home and at Work, https://www.nytimes.com/2018/03/09/realestate/plant-loving-millennia ls-at-home-and-at-work.html

4. Chen Y, Shi Y L, Wang Z Y, Huang L., "Connectivity of wireless sensor networks for plant growth in greenhouse," International Journal of Agricultural and Biological Engineering, vol. 9, no. 1, 2016, pp. 89-98. https://doi.org/10.3965/j.ijabe.201606901.1314

5. Shukla S, Yu C Y, Hardin J D, Jaber F H., "Wireless data acquisition and control systems for agricultural water management projects," Horttechnology, vol. 16, no. 4, 2006, pp. 595-604 https://doi.org/10.21273/HORTTECH.16.4.0595

6. Feng Z G, Lam J, Yang G H., "Optimal partitioning method for stability analysis of continuous/discrete delay systems," International Journal of Robust \& Nonlinear Control, vol. 25, no. 4, 2013, pp. 559-574. https://doi.org/10.1002/rnc.3106

7. Nagaraja, H., Aswani, R., \& Malik, M., "Plant watering autonomous mobile robot," IAES International Journal of Robotics and Automation, vol. 1, no. 3, 2012, pp.

152-162. 
8. Li, F., Ning, T., Shi, L., \& Pang, G., "System Design of the Remote Controlled Intelligent Flowerpo,t" in 3rd Workshop on Advanced Research and Technology in Industry (WARTIA 2017), Atlantis Press, vol. 148, 2017, pp. 310-313. https://doi.org/10.2991/wartia-17.2017.58

9. Zhang, X., Liu, D., Fan, C., Du, J., Meng, F., \& Fang, J., "A novel and smart automatic light-seeking flowerpot for monitoring flower growth environment," International Journal of Agricultural and Biological Engineering, vol. 11, no. 2, 2018, pp. 184-189. https://doi.org/10.25165/j.ijabe.20181102.2786

10. Estrada-Martinez, P. E., \& Garcia-Macias, J. A., "Semantic Interactions in the Internet of Things," International Journal Ad Hoc and Ubiquitous Computing, vol. 9, no. 3/4, 2013, pp. 1-10. https://doi.org/10.1504/IJAHUC.2013.055464

11. Aravind, R., \& Sasipriya, S., "A survey on Hydroponic methods of smart farming and its effectiveness in reducing pesticide usage," International Journal of Pure and Applied Mathematics, vol. 119, no. 12, 2018, pp. 1503-1509.

12. Saini, S., Kumari, P., Yadav, P., Bansal, P., \& Ruhil, N., "Smart farming pot," in 2016 3rd International Conference on Computing for Sustainable Global Development (INDIACom), IEEE, 2016, pp. 3247-3250.

13. Chieochan, O., Saokaew, A., \& Boonchieng, E., "IOT for smart farm: A case study of the Lingzhi mushroom farm at Maejo University," in 2017 14th International Joint Conference on Computer Science and Software Engineering (JCSSE), IEEE, 2017, pp. 1-6.

\section{AUTHORS PROFILE}

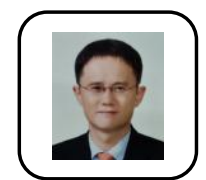

Yeong-Keun Lee Received the B.S. in Business Administration from Yonsei University, Seoul, Rep. of Korea, in 1988. M.S. degree in Mechanical and Automotive Engineering at Kongju National University, Cheonan, Rep. of Korea, in 2017. Ph.D Course in Computer Engineering at Kongju National University, Cheonan, Rep. of Korea. Currently, he is working as a board of chairman at Mirae Corporation. His research interests include Computer Simulation, Smart Farm, Smart Factory, Computer Vision System and IT Convergence.

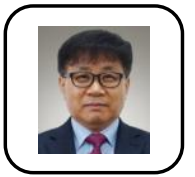

Koo-Rack Park* Received the B.S. in Electronic Engineering from Chung-Ang University, Seoul, Rep. of Korea, in 1986 and the M.S. in Computer Science and engineering from Soongsil University, Seoul, Rep. of Korea, in 1988. He received Ph.D Degree of Computer Science from Kyonggi University, Suwon, Rep. of Korea, in 2000. Currently, he is a Professor in the Department of Computer Science and Engineering at Kongju National University, Cheonan, Rep. of Korea. His research interests include Multimedia, Electronic Commerce, Simulation, Crime Prediction, Predictive Modelling, and IT Convergence.

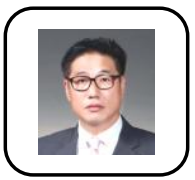

Dong-Hyun Kim Received the B.S. in Electronic Engineering from Chung-Ang University, Seoul, Rep. of Korea, in 1986 and the M.S. degree in Computer Multimedia Engineering from Kongju National University, Gongju, Rep. of Korea, in 2005. He received the Ph.D degree of Computer Engineering in 2010 from the Kongju National University, Cheonan, Rep. of Korea. His research interests include Image Processing System, Geographic Intelligence, Computer Simulation, and Multimedia Application, and IT Convergence. 